(c) American Dairy Science Association, 2006.

\title{
Sources of Variation in Milk Flow Characteristics at Udder and Quarter Levels
}

\author{
V. Tančin, ${ }^{* 1}$ B. Ipema,† P. Hogewerf,† and J. Mačuhováł \\ *Slovak Agricultural Research Authority, Hlohovská 2, SK-949 92 Nitra, Slovakia \\ †Animal Sciences Group Wageningen UR, P.O. Box 65, NL-8200 AB Lelystad, The Netherlands \\ ‡Physiology Weihenstephan, Technical University Munich, D-85350 Freising, Germany
}

\section{ABSTRACT}

The aim of this study was to describe and analyze effects of parity, stage of lactation, milkability (3 groups of cows with differing peak flow rates), time of milking, and quarter position on milk production and milk flow measures at udder and quarter levels. Particular emphasis was put on changes to the decline phase and in duration of overmilking. More than 75,800 quarter milk flow curves and more than 19,300 udder milk flow curves obtained from 38 cows throughout lactation were analyzed. Stage of lactation significantly influenced all studied variables at both udder and quarter levels. At the quarter level, the duration of decline phase and the decline ratio (decline phase as a percentage of milking time) decreased from mo 1 to 2 and then gradually increased as lactation advanced. In contrast, at the udder level, duration of decline phase decreased throughout lactation but beginning at mo 2 , the decline ratio increased as lactation advanced. The duration of the overmilking phase of quarters increased from mo 1 to 3 and then decreased in the course of lactation. Parity did not influence peak and average flow rates, the duration of increase phase, or the decline ratio at either udder or quarter levels. All milk flow measures were higher during morning milking except the duration of increase and decline phases at the quarter level and the duration of increase phase at the udder level. Milk yield and the duration of increase phase were not affected by milkability at either level. Quarters from udders with high milkability had longest duration of decline phase and the shortest overmilking phase. Milkability did not influence duration of the decline phase at the udder level. Quarter position influenced all measured variables of milk yield and milk flow. Rear quarters had significantly higher milk yield, longer time of milking, higher peak, and higher average flow rates than front quarters. Front quarters had shorter duration of increase and decline phases than rear quarters.

Received August 15, 2005.

Accepted October 21, 2005.

${ }^{1}$ Corresponding author: tancin@vuzv.sk
The duration of the overmilking phase was almost double for front quarters. There were also differences in measured flow rates between left or right quarters on respective front or rear positions. Measured characteristics reported in this study may be important in setting default parameters in automated milking systems.

Key words: milk flow pattern, cow, quarter, udder

\section{INTRODUCTION}

Milk production and milk flow characteristics are important economic factors in dairy practice. They are used for animal selection (Miller et al., 1976; Bruckmaier et al., 1995), animal breeding, and monitoring of udder health (Perez-Guzman et al., 1986; Duda, 1995; Naumann et al., 1998). In addition to economical aspects of milk production and monitoring of milking efficiencies for farmers, recording of milk flow is used for evaluation and development of milking machines and in setting parameters for their use (Thomas et al., 1991; Butler et al., 1992; Rasmussen, 1993). Measures of milk flow are also important in studying physiological responses of dairy animals to milking (Zinn et al., 1982; Marnet and McKusick, 2001) or indicating the efficiency of milk ejection (Tančin and Bruckmaier, 2001).

The objective of the milkability evaluation in dairy practice is to improve labor efficiency (Dodenhoff et al., 1999). Therefore, besides selecting of cows for higher milk production, concomitantly higher milk flow is required to optimize duration of milking. However, there is evidence that faster milking cows have higher infection risk for mastitis (Grindal and Hillerton, 1991). However, higher peak milk flow and higher average milk flow are not the only measures that seem to be related to increased risk for mastitis. Somatic cell counts also increased with increasing duration of decline phase (Naumann et al., 1998; Dodenhoff et al., 1999) or with increasing duration of overmilking (Natzke et al., 1978). Moreover, sudden changes in the course of milk flow could indicate problems with udder health; that is, teat injury before milk yield changes occur (important mainly in automatic milking systems where no person is present). Therefore, the more accu- 
rately milk flow can be measured and analyzed, the more closely the effect of milking machine and other conditions during milking can be studied. Intensive and rapid development of new milking machines with partial or full automation offers very sophisticated controlling systems allowing analysis of the milking process to minimize possible adverse effects of machine milking on the animals.

However, recent knowledge shows the importance of recording not only udder milk flow, but also quarter milk flow for faster progress in development of milking technology (Mačuhová et al., 2003), better efficiency of milk removal (Wellnitz et al., 1999; Ipema and Hogewerf, 2002; Weiss et al., 2004), and improvement of udder health (Naumann and Fahr, 2000; Tančin et al., 2002, 2003; Kohler and Kaufmann, 2003). However, due to technical limitations, only limited analysis of quarter milk flows are available at present.

The aim of this study was to describe in detail and to comprehensively analyze effects of parity, stage of lactation, milkability, quarter position on the milk production, and milk flow measures at the udder and quarter levels. Durations of overmilking and of the decline phase related to stage of lactation and milkability were particularly emphasized because those variables are considered risk factors for mastitis.

\section{MATERIALS AND METHODS}

\section{Animals and Milking Procedure}

The study was conducted at the experimental farm "De Vijf Roeden" in the Netherlands in 1999 and 2000. A total of 38 Holstein cows, in their first to sixth lactations, were investigated during 10 mo of lactation. Cows were free of clinical symptoms of mastitis. Cows received a mixed ration consisting of $70 \%$ grass silage and 30\% whole-crop corn silage on a DM basis. Some concentrate was added to the mixture so that it was sufficient for a milk yield of 26 to $27 \mathrm{~kg} / \mathrm{cow}$ per $\mathrm{d}$. Additional concentrate was fed in the milking parlor with a minimum of $1 \mathrm{~kg} / \mathrm{d}$ and more depending on the level of the daily milk yield above $27 \mathrm{~kg}$.

Cows were milked twice a day at 0530 and $1530 \mathrm{~h}$ in a $2 \times 3$ side-opening tandem milking parlor. Premilking udder preparation consisted of forestripping, cleaning, and drying with a dry paper towel for 8 to 10 s per udder. After this short preparation, a cluster was immediately attached. Milking and pulsation vacuum was set at 43 $\mathrm{kPa}$. Pulsation ratio was $65: 35$ at a rate of 60 cycles/ min. Milk was collected in receiver jars mounted in a basement below the milking stalls. The height difference between milking cluster at the udder and the bottom level of the receiver jar was about $180 \mathrm{~cm}$. The cluster (all 4 teat cups) was automatically removed $4 \mathrm{~s}$ after the whole udder milk flow had decreased below $0.3 \mathrm{~kg} / \mathrm{min}$ for a period of $6 \mathrm{~s}$.

\section{Equipment for Milk Flow Measuring}

The quarter milk flow was recorded at every milking for 10 mo of lactation. Each stall in the milking parlor was equipped with 4 milk receiver jars. The advancing weight of each jar was recorded each second. The delay between the time milk left the teat until it was measured in the receiver jar was about $10 \mathrm{~s}$. The milk weight registrations during milking were converted to a milk flow rate profile for each individual quarter (Ipema and Hogewerf, 2002) as described below in detail. The advance of milk yield per quarter during milking is recorded each second (Figure 1a).

In a number of steps, characteristic points of the milk removal process are determined from the recorded milk yield curve. The first derivative of the milk yield curve and some smoothing gives the milk flow curve as shown in Figure 1b.

The second derivative (Figure 1c) gives information about points where milk flow-rate changes approach zero or alter direction. The point where the second derivative approaches zero and stays around the zerolevel for a certain time is defined as the end of the increase phase and the beginning of the steady state phase (plateau). During the first 2 min of the milking process, when an increasing milk flow rate is followed by a decreasing flow rate, that time is defined as a bimodal beginning of the milk removal process. The point when the second derivative changes from zerolevel into negative values for a certain time is defined as the end of the steady state phase and the beginning of the declining phase. During the declining phase, the point when the milk flow rate drops below $0.075 \mathrm{~kg} /$ min during a certain time is defined as the end of the declining phase and beginning of the overmilking phase. The overmilking phase ends when the teat cup is removed (detached) or when the milking vacuum is disconnected from the teat end.

After the characteristic points have been identified, duration of the phases and the amounts of milk produced during each phase can be determined.

Finally, the maximum milk flow rate is defined as the largest increase in milk yield during any 30 -s period. Milking time is defined as the sum of the durations of the increase, plateau, and decline phases. Decline ratio was calculated as the duration of decline phase divided by the duration of milking time multiplied by 100 .

The sum of the advancing milk yields of all 4 quarters gives the milk yield curve for the whole udder. The same procedure as just described for a single quarter is followed for the analysis of milk yield of the total 
a
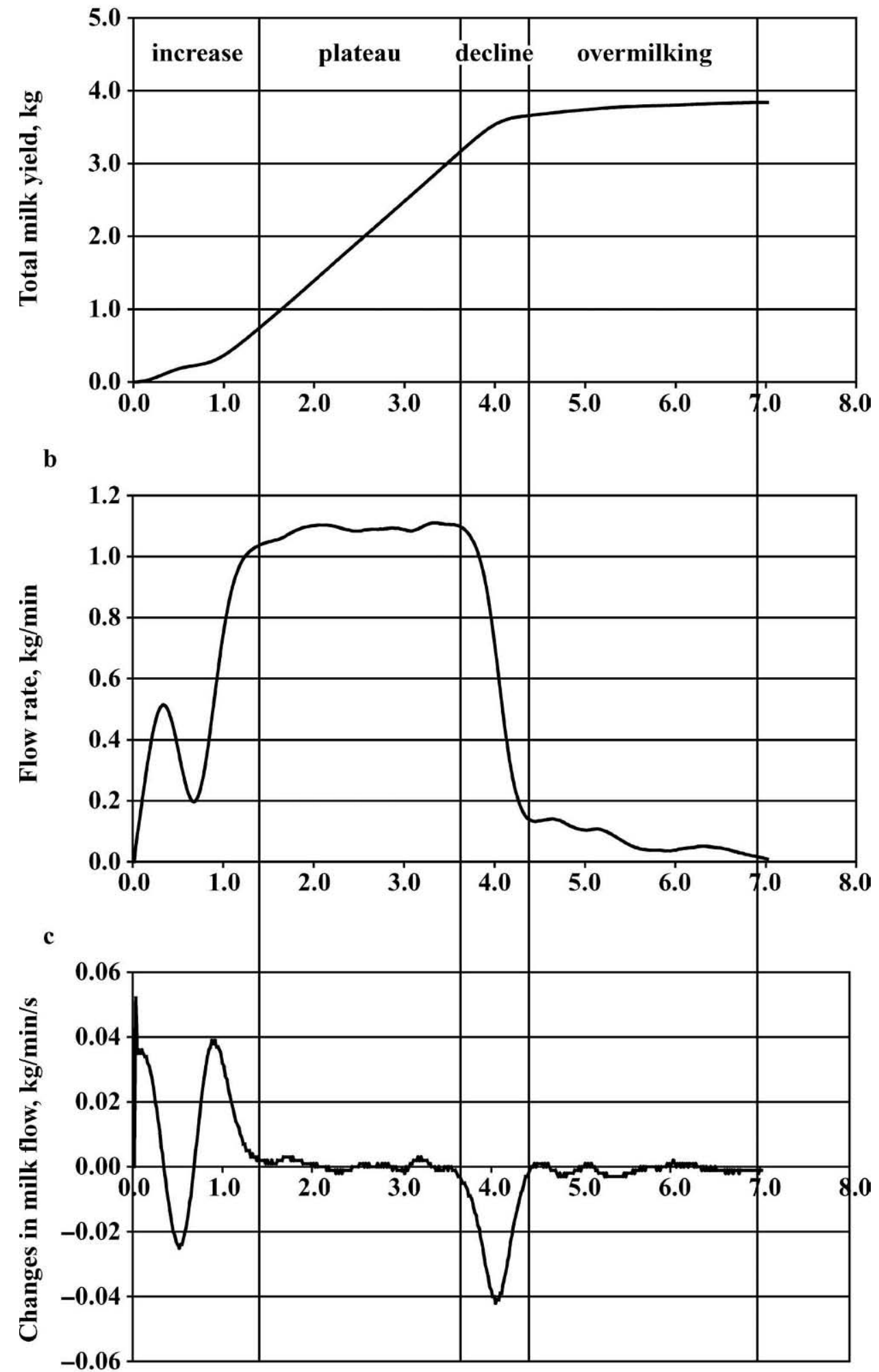

Milking time, min

Figure 1. Description of the single phases of milk flow. 
udder. The only difference is that at the udder level the decreasing phase ends when the teat cups are removed (detached).

\section{Statistical Analyses}

The milk yield and the milk flow measures at quarter and udder level were analyzed by the mixed model as implemented in the SAS package (ver. 8.2; SAS Institute, 2001). Quarters with milk yields lower than 0.9 $\mathrm{kg}(0.8 \%$ of quarters $)$ and udders with milk yields lower than $4.0 \mathrm{~kg}$ ( $0.3 \%$ of udders) were excluded from the statistical evaluation.

In the statistical model for udder data (Model 1 below), the effects of parity, stage of lactation, milkability, and time of milking (morning, evening) were tested. In the model for quarter traits (Model 2 below), an additional factor (position of single quarters) was included.

The stage of lactation was divided into 10 periods representing $10 \mathrm{mo}$ of lactation. To evaluate the effect of stage of lactation in relation to milkability by additional models, at udder level (Model 1a) and quarter levels (Model 2a), stage of lactation was divided into 21 periods of 2 -wk intervals. Parity included 3 groups of cows: primiparous (primi, $\mathrm{n}=13$ ); second-lactation $(\mathrm{n}=15)$; and multiparous (multi, $\geq 3$ lactations, $n=10$ ). The milkability of cows represents 3 groups of cows divided on the basis of the average of maximum udder milk flow rates during the whole 10 mo of lactation; that is, low milkability ( $<3.2 \mathrm{~kg} / \mathrm{min}, \mathrm{n}=13$ cows $)$, middle milkability (between 3.2 and $4.2 \mathrm{~kg} / \mathrm{min}, \mathrm{n}=14$ cows), and high milkability ( $>4.2 \mathrm{~kg} / \mathrm{min}, \mathrm{n}=11$ cows).

Time of milking represents morning and evening milking. Quarter factor represents the positions of individual quarters; that is, front left, front right, rear left, and rear right.

The statistical model can be written in the following form:

\section{Udder data:}

$$
\begin{aligned}
y_{i j k l p}=\mu & +P A R_{i}+S T A G E_{j}+P E A K_{k} \\
& +T I M E_{l}+u_{p}+e_{i j k l p} \\
y_{i n k l p}=\mu & +P A R_{i}+S T A G E W_{n}\left(P E A K_{k}\right) \\
& +T I M E_{l}+u_{p}+e_{i n k l p}
\end{aligned}
$$

\section{Quarter data:}

$$
\begin{gathered}
y_{i j k l m p}=\mu+P A R_{i}+S T A G E_{j}+P E A K_{k} \\
+T I M E_{l}+Q U A R_{m}+u_{p}+e_{i j k l m p}
\end{gathered}
$$

$$
\begin{gathered}
y_{\text {inklmp }}=\mu+P A R_{i}+\operatorname{STAGEW}_{n}\left(\mathrm{PEAK}_{k}\right) \\
+ \text { TIME }_{l}+Q U A R_{m}+u_{p}+e_{\text {inklmp }}
\end{gathered}
$$

where $y_{i j k l p}$ and $y_{i j k l m p}=$ the measurements for milk yield and flow traits at udder and quarter levels, respectively, for Models [1] and [2]; $y_{\text {inklp }}=$ the measurements of the duration of decline phase for Model (1a); $y_{\text {inklmp }}=$ the measurements of the duration of decline and overmilking phase for Model (2a); $\mu$ = overall mean; $\mathrm{PAR}_{\mathrm{i}}=$ the fixed effects of parity ( $i=1,2$, or $3+$ lactations); $\mathrm{STAGE}_{\mathrm{j}}=$ fixed effect of stage of lactation $(\mathrm{j}=1$ to 10 mo); STAGEW $_{\mathrm{n}}=$ fixed effect of stage of lactation $(\mathrm{n}=$ 1 to 212 -wk periods); $\mathrm{STAGEW}_{\mathrm{n}}\left(\mathrm{PEAK}_{\mathrm{k}}\right)=$ fixed effect of stage of lactation nested within milkability ( $\mathrm{n}=1$ to $21, \mathrm{k}=1$ to 3$) ; \mathrm{PEAK}_{\mathrm{k}}=$ fixed effect of milkability $(\mathrm{k}=$ 1 to 3$) ; \mathrm{TIME}_{1}=$ fixed effect of time of milking ( $1=1$ to 2 for a.m. and p.m.); $\mathrm{QUAR}_{\mathrm{m}}=$ fixed effect of quarter positions $(\mathrm{m}=1$ to 4$) ; u_{p}=$ random effect of cow, $u_{p} \sim$ $\mathrm{N}\left(0, \sigma_{\mathrm{c}}^{2}\right)$; and $e_{i j n k l m p}=$ random error, assuming $e_{i j n k l m p}$ $\sim \mathrm{N}\left(0, \mathrm{I} \sigma_{\mathrm{e}}^{2}\right)$.

From the least squares means obtained from Models 1a and 2a, we have calculated correlations in SAS program.

Differences between the levels of the effects were tested by Scheffé's multiple range test for studied traits. Results are presented as least squares means \pm standard error.

\section{RESULTS}

In total, 75,888 quarter milk flow curves and 19,363 udder milk flow curves obtained from 38 cows throughout first 10 mo of lactation were analyzed.

Stage of Lactation. Stage of lactation significantly influenced all studied measures at udder and quarter levels (Tables 1 and 2, respectively). Peak milk production was reached at the second month of lactation, and thereafter, milk production decreased. The values of milking time, duration and yield of plateau phase, and average flow rate corresponded to milk yield changes throughout lactation. Peak flow rates at both udder and quarter levels were relatively stable until mo 7 although there was a significant increase in mo 6 . The peak flow rate gradually reduced as the lactation advanced at both udder and quarter levels. The duration of the increase phase was prolonged at both udder and quarter levels throughout lactation.

At the quarter level, the duration of decline phase and decline ratio decreased from mo 1 to mo 2 and then increased during following stages of lactation. In contrast, at the udder level, duration of decline phase decreased but decline ratio decreased from mo 1 to 2 and then increased throughout lactation (Tables 1 and 


\begin{tabular}{lllllll} 
& & & & Month of lactation ${ }^{1}$ \\
\cline { 2 - 6 } Variable & 1 & 2 & 3 & 4 & 5 &
\end{tabular}

Month of lactation ${ }^{1}$

\begin{tabular}{|c|c|c|c|c|c|c|c|c|c|c|}
\hline Milk yield, kg & $16.36 \pm 0.32^{\mathrm{a}}$ & $19.86 \pm 0.32^{\mathrm{b}}$ & $19.06 \pm 0.32^{\mathrm{c}}$ & $17.69 \pm 0.32^{\mathrm{d}}$ & $16.42 \pm 0.32^{\mathrm{a}}$ & $14.98 \pm 0.32^{\mathrm{e}}$ & $13.59 \pm 0.32^{\mathrm{f}}$ & $12.06 \pm 0.32^{\mathrm{g}}$ & $10.32 \pm 0.32^{\mathrm{h}}$ & $8.62 \pm 0.32^{\mathrm{i}}$ \\
\hline Milking time, s & $435 \pm 9^{\mathrm{a}}$ & $492 \pm 9^{b}$ & $489 \pm 9^{b}$ & $452 \pm 9^{\mathrm{c}}$ & $423 \pm 9^{d}$ & $396 \pm 9^{e}$ & $376 \pm 9^{f}$ & $351 \pm 9^{g}$ & $332 \pm 9^{\mathrm{h}}$ & $320 \pm 9^{i}$ \\
\hline $\begin{array}{l}\text { Peak flow rate, } \\
\mathrm{kg} / \mathrm{min} \\
\text { Average flow }\end{array}$ & $3.77 \pm 0.15^{\mathrm{a}}$ & $3.66 \pm 0.15^{\mathrm{b}}$ & $3.63 \pm 0.15^{\mathrm{c}}$ & $3.67 \pm 0.15^{b}$ & $3.68 \pm 0.15^{b}$ & $3.74 \pm 0.15^{\mathrm{c}}$ & $3.68 \pm 0.15^{b}$ & $3.58 \pm 0.15^{\mathrm{d}}$ & $3.33 \pm 0.15^{\mathrm{e}}$ & $3.02 \pm 0.15^{\mathrm{f}}$ \\
\hline $\begin{array}{l}\text { rate, } \mathrm{kg} / \mathrm{min} \\
\text { Milk yield }\end{array}$ & $2.29 \pm 0.08^{\mathrm{a}}$ & $2.56 \pm 0.08^{b}$ & $2.43 \pm 0.08^{c}$ & $2.43 \pm 0.08^{c}$ & $2.41 \pm 0.08^{\mathrm{c}}$ & $2.32 \pm 0.08^{\mathrm{a}}$ & $2.23 \pm 0.08^{\mathrm{d}}$ & $2.09 \pm 0.08^{\mathrm{e}}$ & $1.87 \pm 0.08^{f}$ & $1.69 \pm 0.08^{\mathrm{g}}$ \\
\hline $\begin{array}{l}\text { in first min, } \mathrm{kg} \\
\text { Duration of milk } \\
\text { flow phase, } \mathrm{s}\end{array}$ & $1.62 \pm 0.06^{\mathrm{a}}$ & $1.59 \pm 0.06^{\mathrm{b}}$ & $1.38 \pm 0.06^{\mathrm{c}}$ & $1.44 \pm 0.06^{\mathrm{d}}$ & $1.38 \pm 0.06^{\mathrm{e}}$ & $1.24 \pm 0.06^{\mathrm{f}}$ & $1.15 \pm 0.06^{\mathrm{g}}$ & $1.04 \pm 0.06^{\mathrm{h}}$ & $0.92 \pm 0.06^{\mathrm{i}}$ & $0.82 \pm 0.06^{\mathrm{j}}$ \\
\hline Increase & $75 \pm 2^{\mathrm{a}}$ & $79 \pm 2^{\mathrm{b}}$ & $82 \pm 2^{\mathrm{c}}$ & $82 \pm 2^{\mathrm{c}}$ & $83 \pm 2^{\mathrm{c}}$ & $87 \pm 2^{\mathrm{d}}$ & $90 \pm 2^{\mathrm{e}}$ & $91 \pm 2^{\mathrm{e}}$ & $92 \pm 2^{\mathrm{f}}$ & $94 \pm 2^{\mathrm{g}}$ \\
\hline Plat & $182 \pm 8^{\mathrm{a}}$ & $252 \pm 2^{\mathrm{b}}$ & $240 \pm 2^{\mathrm{c}}$ & $217 \pm 2^{\mathrm{d}}$ & $192 \pm 2^{\mathrm{e}}$ & $162 \pm 2^{\mathrm{f}}$ & $140 \pm 2^{\mathrm{g}}$ & $120 \pm 2^{\mathrm{h}}$ & $103 \pm 2^{\mathrm{i}}$ & $89 \pm 2^{j}$ \\
\hline Decline & $175 \pm 6^{\mathrm{a}}$ & $167 \pm 6^{b}$ & $163 \pm 6^{c}$ & $152 \pm 6^{\mathrm{d}}$ & $147 \pm 6^{\mathrm{e}}$ & $147 \pm 6^{\mathrm{e}}$ & $147 \pm 6^{\mathrm{e}}$ & $141 \pm 6^{\mathrm{f}}$ & $138 \pm 6^{\mathrm{g}}$ & $137 \pm 6^{\mathrm{g}}$ \\
\hline $\begin{array}{l}\text { Yield per } \\
\text { phase, kg }\end{array}$ & & & & & & & & & & \\
\hline Increase & $2.37 \pm 0.06^{\mathrm{a}}$ & $2.51 \pm 0.06^{\mathrm{b}}$ & $2.52 \pm 0.06^{\mathrm{b}}$ & $2.52 \pm 0.06^{\mathrm{b}}$ & $2.49 \pm 0.06^{\mathrm{b}}$ & $2.51 \pm 0.06^{\mathrm{b}}$ & $2.46 \pm 0.06^{\mathrm{c}}$ & $2.30 \pm 0.06^{\mathrm{d}}$ & $2.13 \pm 0.06^{\mathrm{e}}$ & $1.88 \pm 0.06^{f}$ \\
\hline Plateau & $9.64 \pm 0.31^{\mathrm{a}}$ & $13.17 \pm 0.31^{\mathrm{b}}$ & $14.32 \pm 0.31^{\mathrm{c}}$ & $11.21 \pm 0.31^{\mathrm{d}}$ & $10.09 \pm 0.31^{\mathrm{e}}$ & $8.56 \pm 0.31^{\mathrm{f}}$ & $7.29 \pm 0.31^{\mathrm{g}}$ & $6.14 \pm 0.31^{\mathrm{h}}$ & $4.91 \pm 0.31^{\mathrm{i}}$ & $3.78 \pm 0.31^{\mathrm{j}}$ \\
\hline Decline & $4.31 \pm 0.17^{\mathrm{a}}$ & $4.10 \pm 0.17^{\mathrm{b}}$ & $4.14 \pm 0.17^{\mathrm{b}}$ & $3.91 \pm 0.17^{\mathrm{c}}$ & $3.81 \pm 0.17^{\mathrm{d}}$ & $3.88 \pm 0.17^{\mathrm{c}}$ & $3.78 \pm 0.17^{\mathrm{d}}$ & $3.56 \pm 0.17^{\mathrm{e}}$ & $3.29 \pm 0.17^{\mathrm{f}}$ & $2.92 \pm 0.17^{g}$ \\
\hline Decline ratio, \% & $41 \pm 1^{\mathrm{a}}$ & $32 \pm 1^{b}$ & $34 \pm 1^{\mathrm{c}}$ & $34 \pm 1^{\mathrm{c}}$ & $35 \pm 1^{\mathrm{d}}$ & $37 \pm 1^{\mathrm{e}}$ & $39 \pm 1$ & $40 \pm 1^{\mathrm{g}}$ & $42 \pm 1^{\mathrm{a}}$ & $43 \pm 1^{\mathrm{h}}$ \\
\hline
\end{tabular}

${ }^{a-j}$ Least squares means within a row without a common superscript letter were significantly different at $P<0.05$. Treatment effect for all variables was $P<0.0001$.

${ }^{1}$ Month of lactation represents the first 10 mo of lactation.

Table 2. Least squares means and SEM of measured variables at the quarter level during different stages of lactation

\begin{tabular}{|c|c|c|c|c|c|c|c|c|c|c|}
\hline \multirow[b]{2}{*}{ Variable } & \multicolumn{10}{|c|}{ Month of lactation ${ }^{1}$} \\
\hline & 1 & 2 & 3 & 4 & 5 & 6 & 7 & 8 & 9 & 10 \\
\hline Milk yield, kg & $4.09 \pm 0.08^{a}$ & $4.96 \pm 0.08^{b}$ & $4.76 \pm 0.08^{\mathrm{c}}$ & $4.42 \pm 0.08^{\mathrm{d}}$ & $4.09 \pm 0.08^{\mathrm{a}}$ & $3.74 \pm 0.08^{\mathrm{e}}$ & $3.39 \pm 0.08^{f}$ & $3.01 \pm 0.08^{\mathrm{g}}$ & $2.61 \pm 0.08^{\mathrm{h}}$ & $2.19 \pm 0.08^{\mathrm{i}}$ \\
\hline Milking time, s & $371 \pm 7^{\mathrm{a}}$ & $427 \pm 7^{\mathrm{b}}$ & $423 \pm 7^{\mathrm{b}}$ & $393 \pm 7^{\mathrm{c}}$ & $367 \pm 7^{\mathrm{d}}$ & $343 \pm 7^{\mathrm{e}}$ & $325 \pm 7^{\mathrm{f}}$ & $305 \pm 7^{\mathrm{g}}$ & $288 \pm 7^{\mathrm{h}}$ & $277 \pm 7^{\mathrm{i}}$ \\
\hline Peak flow rate, $\mathrm{kg} / \mathrm{min}$ & $0.98 \pm 0.04^{\mathrm{a}}$ & $0.95 \pm 0.04^{\mathrm{b}}$ & $0.95 \pm 0.04^{b}$ & $0.95 \pm 0.04^{\mathrm{b}}$ & $0.95 \pm 0.04^{\mathrm{b}}$ & $0.96 \pm 0.04^{\mathrm{c}}$ & $0.95 \pm 0.04^{\mathrm{b}}$ & $0.92 \pm 0.04^{\mathrm{d}}$ & $0.86 \pm 0.04^{\mathrm{e}}$ & $0.79 \pm 0.04^{\mathrm{f}}$ \\
\hline Average flow rate, $\mathrm{kg} / \mathrm{min}$ & $0.66 \pm 0.02^{\mathrm{a}}$ & $0.72 \pm 0.02^{\mathrm{b}}$ & $0.70 \pm 0.02^{\mathrm{c}}$ & $0.69 \pm 0.02^{\mathrm{c}}$ & $0.68 \pm 0.02^{\mathrm{d}}$ & $0.62 \pm 0.02^{\mathrm{a}}$ & $0.63 \pm 0.02^{\mathrm{e}}$ & $0.59 \pm 0.02^{\mathrm{f}}$ & $0.54 \pm 0.02^{\mathrm{g}}$ & $0.47 \pm 0.02^{\mathrm{h}}$ \\
\hline Milk yield in first min, $\mathrm{kg}$ & $0.41 \pm 0.01$ & $0.42 \pm 0.01$ & $0.39 \pm 0.01$ & $0.36 \pm 0.01$ & $0.35 \pm 0.01$ & $0.31 \pm 0.01$ & $0.29 \pm 0.01$ & $0.26 \pm 0.01$ & $0.24 \pm 0.01$ & $0.22 \pm 0.01$ \\
\hline \multicolumn{11}{|l|}{ Duration of milk flow } \\
\hline Increase & $66 \pm 2^{\mathrm{a}}$ & $73 \pm 2^{\mathrm{b}}$ & $75 \pm 2^{\mathrm{c}}$ & $76 \pm 2^{\mathrm{c}}$ & $75 \pm 2^{\mathrm{c}}$ & $78 \pm 2^{\mathrm{d}}$ & $79 \pm 2^{\mathrm{e}}$ & $79 \pm 2^{\mathrm{e}}$ & $81 \pm 2^{\mathrm{f}}$ & $82 \pm 2^{\mathrm{f}}$ \\
\hline Plateau & $226 \pm 8^{\mathrm{a}}$ & $302 \pm 8^{\mathrm{b}}$ & $295 \pm 8^{\mathrm{c}}$ & $264 \pm 8^{\mathrm{d}}$ & $238 \pm 8^{\mathrm{e}}$ & $207 \pm 8^{\mathrm{f}}$ & $186 \pm 8^{g}$ & $164 \pm 8^{\mathrm{h}}$ & $143 \pm 8^{\mathrm{i}}$ & $137 \pm 8^{j}$ \\
\hline Decline & $81 \pm 3^{\mathrm{a}}$ & $49 \pm 3^{b}$ & $50 \pm 3^{\mathrm{b}}$ & $52 \pm 3^{c}$ & $54 \pm 3^{\mathrm{d}}$ & $58 \pm 3^{\mathrm{e}}$ & $60 \pm 3^{\mathrm{f}}$ & $63 \pm 3^{\mathrm{f}}$ & $66 \pm 3^{\mathrm{g}}$ & $68 \pm 3^{g}$ \\
\hline Overmilking & $69 \pm 3^{\mathrm{a}}$ & $73 \pm 3^{b}$ & $74 \pm 3^{b}$ & $66 \pm 3^{a}$ & $64 \pm 3^{c}$ & $61 \pm 3^{\mathrm{d}}$ & $59 \pm 3^{\mathrm{e}}$ & $55 \pm 3^{\mathrm{f}}$ & $52 \pm 3^{g}$ & $51 \pm 3^{\mathrm{h}}$ \\
\hline \multicolumn{11}{|l|}{ Yield per phase, kg } \\
\hline Increase & $0.47 \pm 0.01^{\mathrm{a}}$ & $0.54 \pm 0.01^{\mathrm{b}}$ & $0.53 \pm 0.01^{\mathrm{b}}$ & $0.52 \pm 0.01^{\mathrm{c}}$ & $0.51 \pm 0.01^{\mathrm{d}}$ & $0.49 \pm 0.01^{\mathrm{a}}$ & $0.47 \pm 0.01^{\mathrm{e}}$ & $0.43 \pm 0.01^{\mathrm{f}}$ & $0.41 \pm 0.01^{\mathrm{g}}$ & $0.36 \pm 0.01^{\mathrm{h}}$ \\
\hline Plateau & $3.08 \pm 0.08^{\mathrm{a}}$ & $4.02 \pm 0.08^{\mathrm{b}}$ & $3.82 \pm 0.08^{\mathrm{c}}$ & $3.48 \pm 0.08^{\mathrm{d}}$ & $3.17 \pm 0.08^{\mathrm{e}}$ & $2.79 \pm 0.08^{f}$ & $2.46 \pm 0.08^{\mathrm{g}}$ & $2.11 \pm 0.08^{\mathrm{h}}$ & $1.74 \pm 0.08^{\mathrm{i}}$ & $1.42 \pm 0.08^{\mathrm{j}}$ \\
\hline Decline & $0.48 \pm 0.02^{\mathrm{a}}$ & $0.35 \pm 0.02^{\mathrm{b}}$ & $0.36 \pm 0.02^{\mathrm{b}}$ & $0.38 \pm 0.02^{\mathrm{c}}$ & $0.38 \pm 0.02^{\mathrm{c}}$ & $0.42 \pm 0.02^{\mathrm{d}}$ & $0.42 \pm 0.02^{\mathrm{d}}$ & $0.42 \pm 0.02^{\mathrm{d}}$ & $0.42 \pm 0.02^{\mathrm{d}}$ & $0.39 \pm 0.02^{\mathrm{e}}$ \\
\hline Decline ratio, \% & $23 \pm 1^{\mathrm{a}}$ & $12 \pm 1^{\mathrm{b}}$ & $13 \pm 1^{\mathrm{b}}$ & $14 \pm 1^{\mathrm{c}}$ & $16 \pm 1^{\mathrm{d}}$ & $18 \pm 1^{\mathrm{e}}$ & $19 \pm 1^{\mathrm{f}}$ & $21 \pm 1^{\mathrm{g}}$ & $24 \pm 1^{\mathrm{a}}$ & $25 \pm 1^{\mathrm{h}}$ \\
\hline
\end{tabular}

${ }^{\mathrm{a}-\mathrm{j}}$ Least squares means within a row without a common superscript letter were significantly different at $P<0.05$. Treatment effect for all variables was $P<0.0001$.

${ }^{1}$ Month of lactation represents the first 10 mo of lactation. 
Table 3. Least squares means of measured variables related to parity ${ }^{1}$

\begin{tabular}{|c|c|c|c|c|c|c|c|c|}
\hline \multirow[b]{2}{*}{ Variable } & \multicolumn{3}{|c|}{ Quarter level } & \multirow{2}{*}{$\begin{array}{l}\text { Treatment } \\
\text { effect, } P \leq\end{array}$} & \multicolumn{3}{|c|}{ Udder level } & \multirow{2}{*}{$\begin{array}{l}\text { Treatmer } \\
\text { effect, } P\end{array}$} \\
\hline & Primi & Second & Multi & & Primi & Second & Multi & \\
\hline Milk yield, kg & $3.09 \pm 0.16^{\mathrm{a}}$ & $4.05 \pm 0.13^{b}$ & $4.02 \pm 0.15^{b}$ & $<0.0001$ & $12.3 \pm 0.5^{\mathrm{a}}$ & $16.1 \pm 0.4^{\mathrm{b}}$ & $16.1 \pm 0.6^{\mathrm{b}}$ & $<0.0001$ \\
\hline Milking time, $\mathrm{s}$ & $308 \pm 12^{\mathrm{a}}$ & $391 \pm 11^{\mathrm{b}}$ & $362 \pm 14^{\mathrm{b}}$ & $<0.0001$ & $344 \pm 22^{\mathrm{a}}$ & $434 \pm 22^{\mathrm{b}}$ & $421 \pm 22^{\mathrm{ab}}$ & $<0.0001$ \\
\hline Peak flow rate, $\mathrm{kg} / \mathrm{min}$ & $0.88 \pm 0.06$ & $0.90 \pm 0.06$ & $0.99 \pm 0.07$ & 0.4560 & $3.34 \pm 0.31$ & $3.45 \pm 0.28$ & $3.75 \pm 0.31$ & 0.4867 \\
\hline Average flow rate, $\mathrm{kg} / \mathrm{min}$ & $0.59 \pm 0.05$ & $0.64 \pm 0.06$ & $0.68 \pm 0.04$ & 0.2508 & $1.99 \pm 0.13$ & $2.14 \pm 0.12$ & $2.29 \pm 0.15$ & 0.3473 \\
\hline Milk yield in first min, $\mathrm{kg}$ & $0.29 \pm 0.02$ & $0.33 \pm 0.02$ & $0.35 \pm 0.03$ & 0.0783 & $1.13 \pm 0.09^{\mathrm{a}}$ & $1.28 \pm 0.09^{\mathrm{ab}}$ & $1.44 \pm 0.11^{\mathrm{b}}$ & 0.0465 \\
\hline \multicolumn{9}{|l|}{ Duration of milk flow phase, $\mathrm{s}$} \\
\hline Increase & $73 \pm 3$ & $76 \pm 3$ & $80 \pm 3$ & 0.2681 & $83 \pm 3$ & $85 \pm 2$ & $92 \pm 3$ & 0.1036 \\
\hline Plateau & $177 \pm 13^{\mathrm{a}}$ & $251 \pm 12^{\mathrm{b}}$ & $217 \pm 15^{\mathrm{b}}$ & $<0.0001$ & $130 \pm 14^{\mathrm{a}}$ & $181 \pm 13^{\mathrm{b}}$ & $158 \pm 16^{\mathrm{ab}}$ & 0.0022 \\
\hline Decline & $57 \pm 4$ & $56 \pm 6$ & $66 \pm 6$ & 0.2601 & $125 \pm 10^{\mathrm{a}}$ & $156 \pm 9^{b}$ & $159 \pm 11^{b}$ & 0.0210 \\
\hline Overmilking & $52 \pm 5^{\mathrm{a}}$ & $70 \pm 5^{\mathrm{b}}$ & $68 \pm 5^{\mathrm{ab}}$ & 0.0341 & & & & \\
\hline \multicolumn{9}{|l|}{ Yield per phase, kg } \\
\hline Increase & $0.38 \pm 0.03^{\mathrm{a}}$ & $0.47 \pm 0.02^{\mathrm{b}}$ & $0.55 \pm 0.03^{\mathrm{c}}$ & 0.0002 & $2.03 \pm 0.11^{\mathrm{a}}$ & $2.31 \pm 0.10^{\mathrm{b}}$ & $2.76 \pm 0.13^{\mathrm{c}}$ & $<0.0001$ \\
\hline Plateau & $2.29 \pm 0.13^{\mathrm{a}}$ & $3.16 \pm 0.14^{\mathrm{b}}$ & $2.98 \pm 0.16^{\mathrm{b}}$ & $<0.0001$ & $7.23 \pm 0.51^{\mathrm{a}}$ & $9.87 \pm 0.51^{\mathrm{b}}$ & $8.86 \pm 0.52^{\mathrm{b}}$ & 0.0007 \\
\hline Decline & $0.37 \pm 0.03$ & $0.38 \pm 0.02$ & $0.45 \pm 0.03$ & 0.0789 & $3.01 \pm 0.28^{\mathrm{a}}$ & $3.92 \pm 0.27^{\mathrm{b}}$ & $4.23 \pm 0.33^{\mathrm{b}}$ & 0.0084 \\
\hline Decline ratio, \% & $20 \pm 1$ & $17 \pm 1$ & $20 \pm 1$ & 0.2831 & $37 \pm 2$ & $38 \pm 2$ & $40 \pm 2$ & 0.5387 \\
\hline
\end{tabular}

${ }^{\mathrm{a}-\mathrm{c}}$ Least squares means within a row of evaluated level without a common superscript letter were significantly different at $P<0.05$.

${ }^{1}$ Cows were divided in 3 groups: primi (primiparous cows, $\mathrm{n}=13$ ), second (second-lactation cows, $\mathrm{n}=15$ ), and multi (cows at $\geq 3$ lactations, $\mathrm{n}=10$ ).

Table 4. Least squares means of measured variables related to milkability ${ }^{1}$

\begin{tabular}{|c|c|c|c|c|c|c|c|c|}
\hline \multirow[b]{2}{*}{ Variable } & \multicolumn{3}{|c|}{ Milkability at quarter level } & \multirow{2}{*}{$\begin{array}{l}\text { Treatment } \\
\text { effect, } P \leq\end{array}$} & \multicolumn{3}{|c|}{ Milkability at udder level } & \multirow{2}{*}{$\begin{array}{l}\text { Treatment } \\
\text { effect, } P \leq\end{array}$} \\
\hline & High & Middle & Low & & High & Middle & Low & \\
\hline Milk yield, kg & $3.82 \pm 0.14$ & $3.74 \pm 0.15$ & $3.62 \pm 0.16$ & 0.5196 & $15.24 \pm 0.52$ & $14.93 \pm 0.51$ & $14.37 \pm 0.53$ & 0.4985 \\
\hline Milking time, s & $290 \pm 13^{\mathrm{a}}$ & $334 \pm 12^{\mathrm{b}}$ & $431 \pm 12^{\mathrm{c}}$ & $<0.0001$ & $310 \pm 14^{\mathrm{a}}$ & $395 \pm 14^{\mathrm{b}}$ & $473 \pm 14^{\mathrm{c}}$ & $<0.0001$ \\
\hline Milk yield in first min, $\mathrm{kg}$ & $0.38 \pm 0.03^{\mathrm{a}}$ & $0.33 \pm 0.02^{\mathrm{b}}$ & $0.28 \pm 0.02^{\mathrm{c}}$ & $<0.0001$ & $1.62 \pm 0.09^{\mathrm{a}}$ & $1.26 \pm 0.10^{\mathrm{b}}$ & $0.97 \pm 0.09^{c}$ & $<0.0001$ \\
\hline \multicolumn{9}{|l|}{ Duration of milk flow phase, $\mathrm{s}$} \\
\hline Increase & $76 \pm 3$ & $77 \pm 3$ & $76 \pm 4$ & 0.9181 & $86 \pm 3$ & $87 \pm 3$ & $84 \pm 3$ & 0.05368 \\
\hline Plateau & $141 \pm 14^{\mathrm{a}}$ & $196 \pm 14^{\mathrm{b}}$ & $308 \pm 13^{\mathrm{c}}$ & $<0.0001$ & $96 \pm 14^{\mathrm{a}}$ & $137 \pm 16^{\mathrm{a}}$ & $236 \pm 14^{\mathrm{b}}$ & $<0.0001$ \\
\hline Decline & $73 \pm 6^{\mathrm{a}}$ & $62 \pm 6^{\mathrm{a}}$ & $44 \pm 6^{\mathrm{b}}$ & 0.0003 & $134 \pm 9$ & $160 \pm 9$ & $149 \pm 9$ & 0.1865 \\
\hline Overmilking & $52 \pm 5^{\mathrm{a}}$ & $64 \pm 5^{b}$ & $75 \pm 5^{b}$ & 0.0041 & & & & \\
\hline \multicolumn{9}{|l|}{ Yield per phase, kg } \\
\hline Increase & $0.61 \pm 0.02^{\mathrm{a}}$ & $0.47 \pm 0.02^{\mathrm{b}}$ & $0.34 \pm 0.02^{\mathrm{c}}$ & $<0.0001$ & $3.11 \pm 0.11^{\mathrm{a}}$ & $1.67 \pm 0.13^{\mathrm{b}}$ & $2.43 \pm 0.11^{\mathrm{c}}$ & $<0.0001$ \\
\hline Plateau & $2.64 \pm 0.14$ & $2.81 \pm 0.16$ & $2.99 \pm 0.17$ & 0.1245 & $7.99 \pm 0.54^{\mathrm{a}}$ & $8.35 \pm 0.61^{\mathrm{ab}}$ & $9.84 \pm 0.55^{b}$ & 0.0221 \\
\hline Decline & $0.55 \pm 0.04^{\mathrm{a}}$ & $0.42 \pm 0.04^{\mathrm{b}}$ & $0.24 \pm 0.04^{\mathrm{c}}$ & $<0.0001$ & $4.19 \pm 0.29^{\mathrm{a}}$ & $4.15 \pm 0.33^{\mathrm{a}}$ & $2.88 \pm 0.29^{b}$ & 0.0011 \\
\hline Decline ratio, $\%$ & $25 \pm 2^{\mathrm{a}}$ & $19 \pm 2^{b}$ & $11 \pm 2^{\mathrm{c}}$ & 0.03731 & $40 \pm 2^{\mathrm{a}}$ & $40 \pm 2^{\mathrm{a}}$ & $32 \pm 2^{b}$ & 0.0002 \\
\hline
\end{tabular}

ఠ ${ }^{a-c}$ Least squares means within a row of evaluated level without a common superscript letter were significantly different at $P<0.05$.

을 $\quad{ }^{1}$ Milkability of cows was divided into 3 groups based on average of maximum udder milk flow during lactation in low ( $<3.2 \mathrm{~kg} / \mathrm{min}$ ), middle (between 3.2 and $4.2 \mathrm{~kg} / \mathrm{min}$ ), and high $(>4.2 \mathrm{~kg} / \mathrm{min})$. 
2). Milk yield of the decline phase at the quarter level was highest at $1 \mathrm{mo}$, lowest at $2 \mathrm{mo}$ but tended to increase as lactation beyond 2 mo, whereas, at the udder level, milk yield of the decline phase decreased by $32 \%$ from 1 mo through 10 mo of lactation. The duration of overmilking phase of quarters increased from the mo 1 to 3 and then decreased in the course of lactation.

Parity Effect. Multiparous and second-lactation cows had significantly higher milk yield than did primiparous cows. Parity did not influence peak and average flow rates, duration of increase phase, or decline ratio at the udder and quarter levels (Table 3). At the quarter level, parity did not influence milk yield in the first minute nor the duration or milk yield of decline phases.

Milkability. Milk yield and the duration of increase phase were not affected by milkability at either level (Table 4). Furthermore, milkability did not influence the duration of decline phase at the udder level and the milk yield of plateau phase at quarter level. Quarters from udders with high milkability had the longest duration of decline phase and shortest overmilking phase.

Stage of Lactation and Milkability. Figure 2 shows a more detailed analysis of the effect of stage of lactation (2-wk intervals) and milkability on the duration of the decline and overmilking phases at quarter level and on the duration of decline phase at udder level. There was a higher correlation between the durations of the decline phase at the quarter level and udder level in cows with high milkability $(\mathrm{r}=0.876, P=0.0001)$ than in cows with middle $(\mathrm{r}=0.549, P=0.01)$, or low milkability ( $\mathrm{r}=-0.144, P=0.532)$. In contrast, the correlation between duration of overmilking at quarter level and decline phase at udder level was lower in cows with high milkability $(\mathrm{r}=0.522, P=0.015)$ than in cows with middle ( $\mathrm{r}=0.787, P=0.0001)$, or low milkability ( $\mathrm{r}=0.747, P=0.0001)$.

Time of Milking. The time of milking (morning and evening milking) significantly influenced all studied measures at both udder and quarter levels (Table 5). All measures were higher during morning milking except the duration of increase and decline phases and the milk yield of decline phase at the quarter level and the duration of increase phase at the udder level.

Quarter Position. Quarter position influenced all measured characteristics of milk yield and milk flow (Table 5). Rear quarters had significantly higher milk yield, longer milking time, and higher peak and average flow rate than front quarters. Front quarters had shorter duration of increase and decline phases than did rear quarters. The duration of the overmilking phase was almost double for front quarters. There were also differences in some measures between left or right quarters on the respective front or rear positions (Table 5).

\section{DISCUSSION}

Although there are many studies about milk flow in dairy cows, most of them are based on data at the udder level. Only a few studies were based on evaluation of quarter milk flow patterns. Our contribution seems to be the first article comprehensively describing variation of both udder and quarter milk flow measures in the same cows throughout lactation.

The effects of the stage of lactation on milk yield and milking time at the quarter and udder levels were similar to those published by others (Rothschild et al., 1980; Zinn et al., 1982; Bruckmaier et al., 1995). Milk yield reached maximum in the second month of lactation and then continuously decreased. Consequently, milking time was the longest in the second month and decreased as lactation proceeded. However, the decrease in milk yield was about $57 \%$ below peak milk yield, whereas the reduction in milking time was only about $35 \%$ below milking time at peak lactation. This supports the idea that milk flow characteristics, or at least some of them, have to vary during lactation.

The average flow rate significantly decreased in the course of lactation at the udder and quarter levels. However, our results for peak flow rate throughout lactation differ from a previous study (Zinn et al., 1982). Peak flow rate was significantly influenced by stage of lactation with numerically higher decreases in the last 2 mo of lactation. There was a significant increase of peak flow during mo 6 of lactation at both levels. We assume that in some dairy cows, high milk production during the first months of lactation could limit free movement of milk through ducts from alveoli to cistern due to the pressure of milk on surrounding tissue. Full udder cisterns may limit the milk ejection efficiency (Mayer et al., 1991).

Reduction in degree of quarter filling during lactation and thus, different lengths of time to reach maximum intramammary pressure (i.e., the time to reach full milk ejection) could cause a significant decrease of peak flow rate during last 2 mo of lactation. With a decreasing degree of udder/quarter filling, there is an increase in the delay from the start of milking until commencement of milk ejection (Bruckmaier and Hilger, 2001). Moreover, by the same duration of udder premilking preparation in our study, a reduced peak flow rate during the last 2 mo could be observed. It could indicate that at the end of lactation, milk was removed from the udder quicker than milk was ejected into udder cisterns from alveoli. Lower udder filling also prolonged the duration of increase phase in the course of lactation. We 

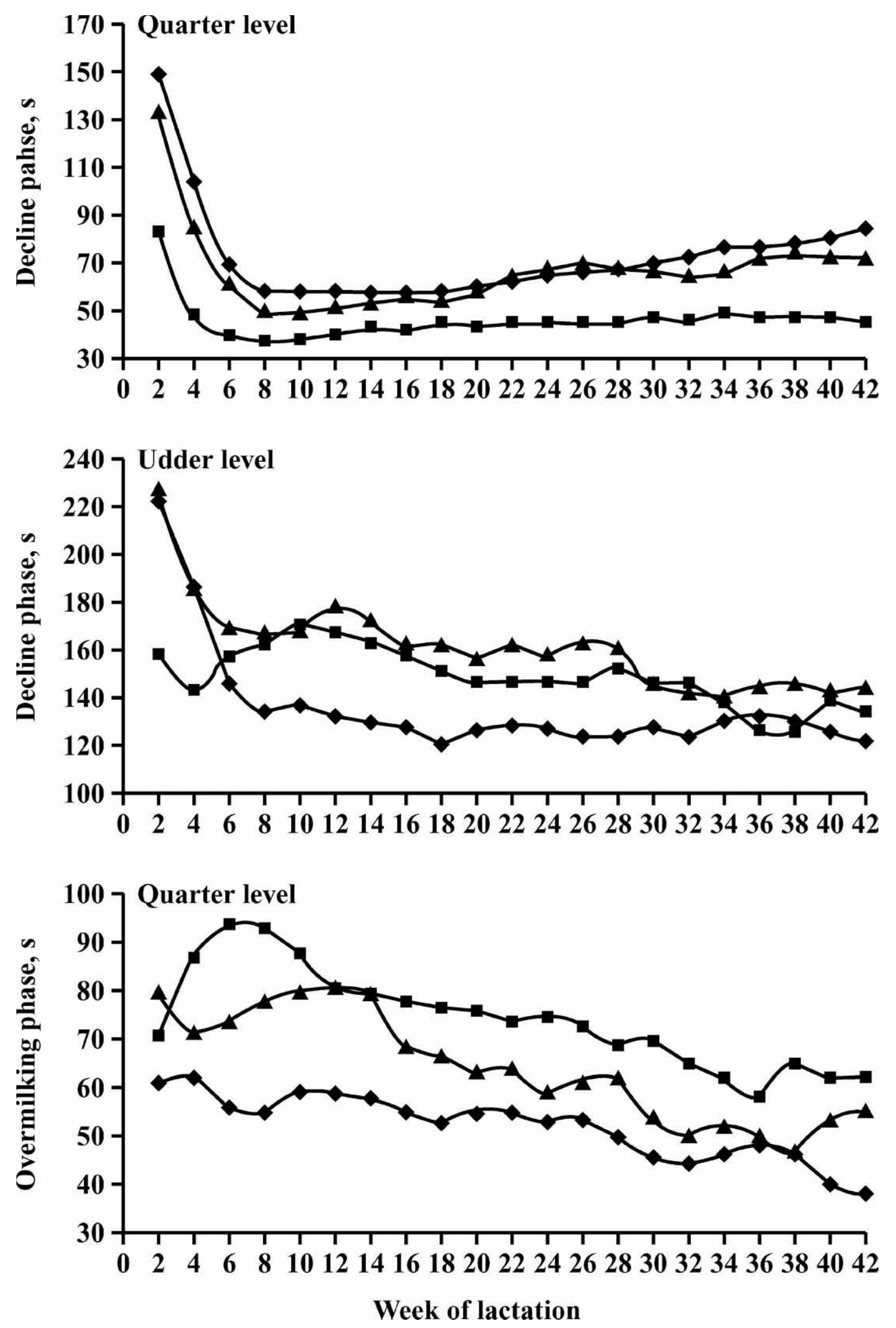

Figure 2. The effect of the stage of lactation (2-wk intervals) on the duration of decline and overmilking phases at quarter levels and decline phase at udder level in relation to the milkability of cows. Milkability was divided by average of maximum udder milk flow during lactation into low $(\boldsymbol{\square} ;<3.2 \mathrm{~kg} / \mathrm{min})$, middle ( $\boldsymbol{\Delta}$; between 3.2 and $4.2 \mathrm{~kg} / \mathrm{min}$ ) and high $(\bullet ;>4.2 \mathrm{~kg} / \mathrm{min})$ milkability groups. 


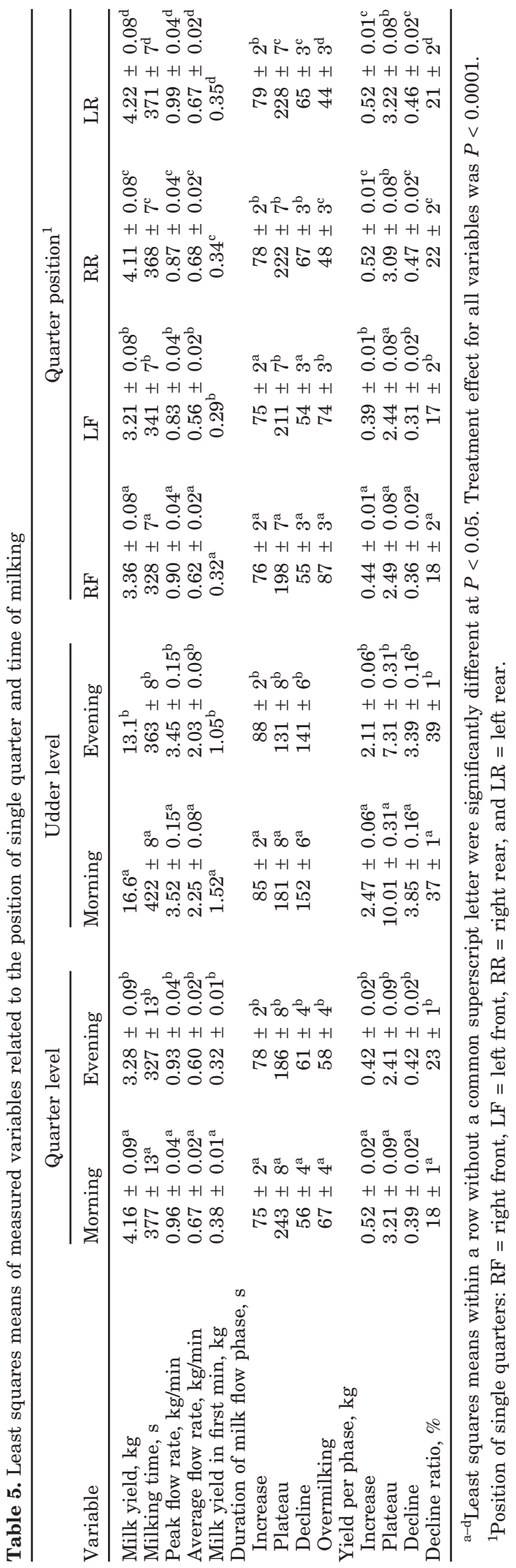

have demonstrated that in the course of lactation, the duration of increase phase increased but amount of milk obtained reduced. Because of short udder preparation by the milker, the main part of milk ejection reflex developed after cluster attachment, as documented by Mačuhová et al. (2003), and was not due to the decreasing sensitivity of milk ejection reflex throughout lactation as suggested previously (Gorewit et al., 1983).

At the udder level, the decline phase mainly begins when milk flow from one or more quarters stopped. In contrast, at the quarter level, the decline phase starts when cisternal filling by alveolar milk is lower than intensity of milk removal. There were also differences in the duration of the decline phase between quarter and udder milk flow throughout lactation. The duration of decline phase at the udder level decreased during lactation, which could explain the reduction of overmilking phase at the quarter level. On the other hand, the duration of the decline phase at the quarter level decreased from mo 1 to 2 of lactation and then increased slowly again. The reason for the longer duration of decline phase of quarters at the beginning and end of lactation is not easy to explain but could be related to cistern filling. At least in primiparous cows, the explanations for the beginning of lactation could be related to milk removal disturbances induced by the adaptation of cows to milking (Tančin and Bruckmaier, 2001) and for the increases during lactation more due to reduced milk production (Bruckmaier and Hilger, 2001). An important factor for the duration of decline phase at the quarter level seems to be associated with the readiness of cows for milking (Wellnitz et al., 1999). That study showed that milking without stimulation prolonged the duration of both increase and decline phases at the quarter level. We have also found that milk yield obtained during the quarter decline phase was relatively stable compared with the reduction of milk yield at the udder level of decline phase or the reduction of milk yield of increase phase at both udder and quarter levels during lactation. Thus, having cows well prepared for milking seems to be an important factor in reducing the negative effect of lowered udder filling in the course of lactation.

The differences between morning and evening milkings were caused by the different time schedule of milking. Different intervals between the 2 milkings, reflected in milk yield obtained at the morning and the evening milking, accounted for the differences in all observed measures. All measures were higher during the morning milking except the duration of increase phase at both levels and the duration and yield of decline phase at quarter level. Peak flow rate was reduced slightly, but significantly, at the evening milking at both levels, as also found by Wagner and Ruegg (2002) 
for udder peak flow. Our results show that unequal intervals between 2 milkings modified the milk flow profiles during the morning and the evening milkings. This seems to be dependent on actual degree of quarter filling, which modified start and the course of milk ejection in single quarters. This could be seen as longer increase and higher decline ratios at the both udder and quarter level and longer decline phase only at the quarter level at evening milking compared with the morning milking.

Milkability is known to be more related to the breed effect and readiness of cows for milking than milk production within breeds (Bruckmaier et al., 1995; Wellnitz et al., 1999). Milkability did not influence milk yield in our study. However, milkability clearly influenced the pattern of milk flow from the udder and the quarter. With increasing milkability, the duration of the decline phase was prolonged and the overmilking phase was reduced at the quarter level; the differences were not significant at the udder level. We have reported similar effects of milkability on decline phase at quarter or udder levels (Tančin et al., 2002, 2003). Recently, similar data were reported by Weiss et al. (2004). Naumann and Fahr (2000) found the longest duration of decline phase from teats with the shortest canal length that also had the highest milk flow. From the correlation data, we could demonstrate that the duration of decline phase of udder milk flow of cows with high milkability depended more on the duration of decline phase at quarter levels compared with cows at moderate or low milkability.

Quarter position influenced all measurements of milk yield and milk flow as described by other researchers (Rothschild et al., 1980). As is well known, the rear quarters had significantly more milk yield, longer milking time, higher peak flow, and higher average flow rate than front quarters. Shorter duration of increase and decline phases were observed in front than in rear quarters as shown in our earlier studies (Tančin et al., 2002, 2003). The duration of overmilking phase was almost double for front quarters, which is similar to other results (Mačuhová et al., 2003).

An interesting issue of this study is the dynamic of the duration of overmilking and the duration of decline phase at both levels in a critical period of lactation and important factors related to higher risk for mastitis. Our previous studies have shown a positive relationship of the duration of decline but not the overmilking phase of quarter milk flow to somatic cell counts (Tančin et al., 2002, 2003). Moreover, the duration of decline phase seems to be more important at the single quarter than at the udder level, if the physiological response of cow to milking is considered. The longer duration of decline phase was found at the beginning and end of lactation, in quarters with high peak flow rate, and in rear quarters. Mentioned factors are mainly connected with higher incidence of udder health problems (Barkema et al., 1999; Grindal and Hillerton, 1991; Rupp and Boichard, 1999). The possible relationship of decline phase at quarter level and somatic cell counts is difficult to explain. Because the majority of machine-induced infections occur near the end of milking, the possible explanation could be adapted from Philpot and Nickerson (1991). They discussed the possible importance of reducing milk flow near the end of milking, which reduces the chance of the microorganisms being flushed out of the teat, and an infection of the quarter may result.

The most frequently discussed negative effect of the duration of overmilking phase on udder health (Natzke et al., 1978; Naumann et al., 1998) seems to have less importance (Wellnitz et al., 1999). Furthermore, although front quarters are often overmilked for longer, they have subclinical or clinical mastitis less frequently than do rear quarters. Our data also showed that quarters in a group of cows with high milkability are overmilked significantly less than quarters from the other 2 milkability groups. Osteras and Lund (1988) reported that overmilking for $>1$ min predisposes cows to subclinical mastitis under field conditions. Therefore, the negative effect of overmilking on udder health has still to be considered.

Reductions of the duration of decrease and overmilking phases seem to be important for good milking practice related to the efficiency of milk removal and udder health. Overmilking is reduced with the use of an automatic cluster device but it could be eliminated by controlling of the end of milking at quarter level, as may be possible in robotic milking. However, no machine devices are presently suitable for reducing the duration of the decline phase, even if the duration of decline phase at quarter level could be considered a critical point in mastitis incidence. Perhaps the modulation of vacuum (Ipema et al., 2005) and better cow preparation to milking (Wellnitz et al., 1999) could be important factors in reducing the duration of the decline phase.

\section{CONCLUSIONS}

We have comprehensively described variation of both udder and quarter milk flow characteristics in the same cows throughout whole lactations. Stage of lactation and time of milking (morning/evening) significantly influenced all measures studied. Milkability and quarter position are important factors influencing milk flow patterns. The duration of the decline phase at the quarter level seems to be an important variable in the physiological response of dairy cows to milking machines. The 
longest duration of decline phase was found at the beginning and end of lactation, in quarters with high peak flow rate, and in rear quarters. This finding could be considered a potential critical point in mastitis incidence. Moreover, cow preparation for milking (milk ejection occurrence causing sufficient cisternal filling by alveolar milk) and vacuum modification could play a role in the duration of decline phase at quarter level.

\section{REFERENCES}

Barkema, H. W., H. A. Deluyker, Y. H. Schukken, and T. J. G. M. Lam. 1999. Quarter-milk somatic cell count at calving and at the first six milkings after calving. Prev. Vet. Med. 38:1-9.

Bruckmaier, R. M., and M. Hilger. 2001. Milk ejection in dairy cows at different degrees of udder filling. J. Dairy Res. 68:369-376.

Bruckmaier, R. M., E. Rothenanger, and J. W. Blum. 1995. Milking characteristics in dairy cows of different breeds from different farms and during the course of lactation. J. Anim. Breed. Genet. 12:293-302.

Butler, M. C., J. E. Hillerton, and R. J. Grindal. 1992. The control of milk flow through the teats of dairy cows. J. Dairy Sci. 75:1019-1024.

Dodenhoff, J., D. Sprengel, J. Duda, and L. Dempfle. 1999. Studies on genetic evaluation of udder health using the LactoCorder. Zuchtungskunde 71:459-472.

Duda, J. 1995. Associations between milkability and susceptibility to mastitis. Zuchtungskunde 67:467-476.

Gorewit, R. C., E. A. Wachs, R. Sagi, and W. G. Merrill. 1983. Current concepts on the role of oxytocin in milk ejection. J. Dairy Sci. 66:2236-2250.

Grindal, R. J., and J. E. Hillerton. 1991. Influence of milk flow rate on new intramammary infection in dairy cows. J. Dairy Res. 58:263-268.

Ipema, B., and P. Hogewerf. 2002. Detachment criteria and milking duration. Pages 33-44 in Proc. 1st North Am. Conf. Robotic milking, Toronto, Canada. Wageningen Pers., Wageningen, The Netherlands.

Ipema, B., V. Tančin, and P. Hogewerf. 2005. Responses of milk removal characteristics of single quarters on different vacuum levels. Pages 49-55 in Proc. Physiol. Tech. Aspects of Machine Milking, ICAR Technical series No. 10, Nitra, Slovak Republic. ICAR, Rome, Italy.

Kohler, S. D., and O. Kaufmann. 2003. Quarter-related measurements of milkings and milk parameters in an AMS.herd. Milchwissenschaft 58:3-6.

Mačuhová, J., V. Tančin, and R. M. Bruckmaier. 2003. Oxytocin release, milk ejection and milk removal in a multi-box automatic milking system. Livest. Prod. Sci. 81:139-147.

Marnet, P. G., and B. C. McKusick. 2001. Regulation of milk ejection and milkability in small ruminants. Livest. Prod. Sci. 70:125-133.

Mayer, H., R. M. Bruckmaier, and D. Schams. 1991. Lactational changes in oxytocin release, intramammary pressure and milking characteristics in dairy cows. J. Dairy Res. 58:159-169.
Miller, R. H., R. E. Pearson, B. T. Weinland, and L. A. Fulton. 1976. Genetic parameters of several measures of milk flow rate and milking time. J. Dairy Sci. 59:957-964.

Natzke, R. P., P. A. Oltenacu, and G. H. Schmidt. 1978. Change in udder health with overmilking. J. Dairy Sci. 61:233-238.

Naumann, I., and R. D. Fahr. 2000. Investigation of milk flow from udder quarters. Arch. Tierz. Dummerstorf 43:431-440.

Naumann, I., R. D. Fahr, and G. Lengerken. 1998. Relationship between somatic cell count of milk and special parameters of milk flow curves of cows. Arch. Tierz. Dummerstorf 41:237-250.

Osteras, O., and A. Lund. 1988. Epidemiological analyses of the associations between bovine udder health and milking machine and milking management. Prev. Vet. Med. 6:91-108.

Perez-Guzman, M. D., J. Claus, W. Junge, and E. Kalm. 1986. Studies on milkability and udder health in cattle. I. Verifying the relationship between milk flow and udder health. Zuchtungskunde 58:21-31.

Philpot, W. N., and S. C. Nickerson. 1991. Mastitis: Counter attack. A strategy to combat mastitis. Babson Bros. Co., Naperville, IL.

Rasmussen, M. D. 1993. Influence of switch level of automatic cluster removers on milking performance and udder health. J. Dairy Res. 60:287-297.

Rothschild, M. F., G. W. Bodoh, R. E. Pearson, and R. H. Miller. 1980. Source of variation in quarter milk flow measures. J. Dairy Sci. 63:1138-1144.

Rupp, R., and D. Boichard. 1999. Genetic parameters for clinical mastitis, somatic cell score, production, udder type traits, and milking ease in first lactation Holsteins. J. Dairy Sci. 82:21982204.

SAS Institute. 2001. Version 8.2. SAS Institute Inc., Cary, NC.

Tančin, V., and R. M. Bruckmaier. 2001. Factors affecting milk ejection and removal during milking and suckling of dairy cows. Vet. Med. Czech. 46:108-118.

Tančin, V., B. Ipema, P. Hogewerf, P. Groot Koerkamp, S. Mihina, and R. M. Bruckmaier. 2002. Milk flow patterns at the end of milking at the whole udder or quarter levels: Relationship to somatic cell counts. Milchwissenschaft 57:306-309.

Tančin, V., B. Ipema, D. Peskovicova, P. Hogewerf, and J. Macuhova. 2003. Quarter milk flow patterns in dairy cows: Factors involved and repeatability. Vet. Med. Czech. 48:275-282.

Thomas, C. V., D. K. Force, D. H. Bremel, and S. Strasser. 1991. Effects of pulsation ratio, pulsation rate, and teatcup liner design on milking rate and milk production. J. Dairy Sci. 74:1243-1249.

Wagner, A. M., and P. L. Ruegg. 2002. The effect of manual forestripping on milking performance of Holstein dairy cows. J. Dairy Sci. 85:804-809.

Weiss D., M. Weinfurtner, and R. M. Bruckmaier. 2004. Teat anatomy and its relationship with quarter and udder milk flow characteristics in dairy cows. J. Dairy Sci. 87:3280-3289.

Wellnitz, O., R. M. Bruckmaier, and J. W. Blum. 1999. Milk ejection and milk removal of single quarters in high yielding dairy cows. Milchwissenschaft 54:303-306.

Zinn, S. A., R. C. Gorewit, and R. Sagi. 1982. Milking response of cows given premilking stimulation at four lactational stages. J. Dairy Sci. 65:668-671. 